\title{
Field emission properties of fractal surfaces
}

\author{
Thiago A. de Assis* \\ Departamento de Química, Universidad Autónoma de Madrid, Cantoblanco, 28049 Madrid, Spain \\ and Departamento de Física y Mecánica, Grupo de Sistemas Complejos, ETSI Agrónomos, Universidad Politécnica de Madrid, Ciudad \\ Universitaria, 28040 Madrid, Spain \\ F. Borondo \\ Departamento de Química and Instituto Mixto de Ciencias Matemáticas CSIC-UAM-UC3M-UCM, Universidad Autónoma de Madrid, \\ Cantoblanco, 28049 Madrid, Spain \\ R. M. Benito \\ Departamento de Física y Mecánica, Grupo de Sistemas Complejos, ETSI Agrónomos, Universidad Politécnica de Madrid, \\ Ciudad Universitaria, 28040 Madrid, Spain \\ R. F. S. Andrade \\ Instituto de Física, Universidade Federal da Bahia, Campus Universitário da Federação, 40210-340, Salvador, BA, Brazil
}

(Received 7 July 2008; revised manuscript received 21 October 2008; published 19 December 2008)

\begin{abstract}
The density of emission current by a rough surface with fractal structure is analyzed. A possible experimental setup is modeled by an irregular emitting surface, iteratively generated by a fractional Brownian motion (FBM) algorithm with roughness (or Hurst) exponent $H=0.3$ and a far away plane representing the anode. The boundaries are held at a fixed potential difference and the Laplace's equation, with lateral periodic conditions, was numerically solved. The solution for the potential leads to the evaluation of the local field and, subsequently, the current density evaluated by using the Fowler-Nordheim's approach. The results hint at a strong local dependence of the electric field with small variations in the roughness of the irregular boundary. The value of the turn-on electric field is found to be $\sim 8 \times 10^{6} \mathrm{~V} / \mathrm{cm}$. Finally, Fowler-plot curves make possible to discuss the connection between the field amplification factor and geometric properties of the emitter surfaces.
\end{abstract}

DOI: $10.1103 /$ PhysRevB.78.235427

PACS number(s): 73.90.+f, 05.45.Df, 61.43.Hv

\section{INTRODUCTION}

Cold field emitters have attracted a great deal of attention since they are good candidates for compact and controllable electron sources, which are important elements in vacuum microelectronic and nanoelectronic devices. ${ }^{1}$ Since some applications require strong electric currents under low voltages, investigations of low work-function materials or structures are of great practical importance. The next generation of such electron emitters requires a fine tuning of several parameters such as material work function, surface structure, field strength, and temperature, in order to warrant that most part of the emission originates from electronic energy levels in the vicinity of the potential barrier. Apart from the issue of evaluating the electronic levels, there subsist several difficulties for the emission problem that arise from classical electrostatic issues related to field emission. Therefore, the accurate description of the electrostatic field with the inhomogeneous geometry of the surface is becoming a timely and very relevant research topics for the theory of field emission by very small scale devices.

Nanometer size metals and semiconductors have good emission properties and also the necessary reproducibility for large scale production. As an example, diamond and carbon nanotube cold cathodes are able to emit electrons at very low electric fields. ${ }^{2}$ This effect results from the geometric fieldenhancement function, which is due to small variations in the emitter shape or in the emitter surroundings, having a strong influence on the produced current. Some results ${ }^{3,4}$ suggest that a substantial reduction in the emitting field can be achieved by increasing the self-similarity of the cathode surface that can be described by a fractal structure. Bonard et $a .^{5}$ measured the field emission of individual carbon nanotube and nanowire emitters by scanning electron microscopy (SEM), concluding that the nanotube geometry and its distance to the anode are important factors for field emission. In addition, several measures on single silicon emitters and silicon emitter arrays reveal that the turn-on field of an individual emitter is much higher than that of an emitter array consisting of several hundreds of nearly identical units.

On the theoretical side, some $a b$ initio calculation results have been reported for the field emitting properties of a carbon linear chain with work function of the order of a few eV. Bianchetti et al. ${ }^{6}$ used density-functional theory (DFT) in the presence of a constant electric field to show that chain polarization has important effects in the electron emission. In a related work, Araidai et ll. $^{7}$ also used DFT in diamond surfaces with subsurface hydrogen substitutional defects. The results show that the ionization energy of hydrogen atoms is small, suggesting that the large experimentally observed emission currents by diamond surfaces are due to the presence of structural irregularities.

In previous works, ${ }^{8,9}$ some of us explored the scaling behavior of equipotential surfaces in an electric field generated by conductors with fractal geometry. The results were analyzed for models of $D+1$ dimensions, with $D=1,2$. In Ref. 8 the influence of the self-affine conductor in the roughness exponent quantifying the irregularities of equipotential pro- 
files was investigated. Afterward, ${ }^{9}$ the average electric-field dependence on the boundary roughness was studied. In both contributions, the scaling properties of both equipotential profiles and surfaces close to the boundary reveal fractal properties which correlate with those of the basal surface.

The purpose of this work is to present a theoretical analysis of the influence of the fractal structure of cold emitted conductors on the emission properties. We discuss the connection between the geometrical properties of the emitter surface, fractal dimension $d_{f}$, roughness $W$, and the local intensity of electric field. In our opinion, the consideration of these issues can contribute to improve the understanding of two specific points: (i) how the surface fractal dimension and protuberances affect the emission, and (ii) how the presence of irregularities in the metal corrugated surfaces at nanometric scale influences the electron emission density by an effective-field amplification.

The organization of the paper is as follows. We start by modeling the irregular cathode by self-affine surfaces provided by the fractional Brownian motion (FBM) algorithm, and solving Laplace's equation for the electrostatic problem in $2+1$ dimensions. Periodic conditions are considered along the $x$ and $y$ directions. The emission current follows from Fowler-Nordheim (FN) approach, while the evaluation of the roughness exponent is done by using the root-mean-square (rms) methodology. ${ }^{10}$ In Sec. II, the theoretical framework of the methods used for generating the self-affine surfaces and the field emission calculations are presented. Section III is devoted to the presentation and discussion of results, thus providing a connection between the geometric features of irregular surface emitter and the current density. Finally, we conclude in Sec. IV by summarizing our main conclusions.

\section{METHODOLOGY}

\section{A. FBM Model}

FBM algorithms ${ }^{11}$ generate random self-affine objects with a specific roughness exponent. In one dimension, this leads to a random sequence, $\bar{z}(t)$, of a certain parameter $t$. The variable $\bar{z}$ has Gaussian distribution increments, $\bar{z}\left(t_{2}\right)$ $-\bar{z}\left(t_{1}\right)$, its variance being proportional to $\left(t_{2}-t_{1}\right)^{2 H}$ with 0 $<H<1$. This means that, starting at $t_{0}=0$ with $\bar{z}\left(t_{0}\right)=0$, the two functions $\bar{z}(r t)$ and $r^{H} \bar{z}(t)$, where $r$ is an scale parameter, are statistically undistinguishable. For any given $\bar{z}_{0}$, the set of values of $t$ satisfying $\bar{z}(t)=\bar{z}_{0}$ are points on a self-affine fractal of dimension $d_{f}=1-H$.

Any FBM algorithm can be generalized to higher dimensions, with a multidimensional process $\bar{z}\left(t_{1}, t_{2}, \ldots, t_{n}\right)$, where all directions are statistically equivalent. In this work, the self-affine sets are constructed by the FBM midpoint displacement algorithm. ${ }^{12}$ This corresponds to a FBM in a limited interval $[0,1]$, which can be later conveniently rescaled to the size of the system we intend to model. The method starts by fixing the values of $\bar{z}$ at the interval end points, i.e., $\bar{z}_{0}=0$ and $\bar{z}_{1}$, where $\bar{z}_{1}$ is obtained by a randomly process with a given variance $\varrho^{2}$. Next, the interval is further partitioned into two subintervals, and the midpoint value $\bar{z}(0.5)$ is calculated as the average between $\bar{z}_{0}$ and $\bar{z}_{1}$ plus a random displacement $\eta_{1}$. That is

$$
\bar{z}(0.5)=0.5\left(\bar{z}_{0}+\bar{z}_{1}\right)+\eta_{1} .
$$

Displacement $\eta_{1}$ is a random Gaussian variable with variance $\delta_{1}^{2} \sim \varrho^{2} / 2^{2 H}$. In this way, the profile Hurst exponent, $H$, is a parameter of the generating algorithm. The same iterative process is repeated for each new interval, $i$, of the generation $n$, i.e., we successively evaluate the displacements $\eta_{n, i}, i=1, \ldots, 2^{n}$, with the help of Eq. (1), where variances $\delta_{n}^{2}$ are proportional to $\varrho^{2} / 2^{2 n H}$ for generation $n$, while the value of $H$ is kept constant.

In this work, we consider the situation in which $\bar{z}(x, y)$ $\equiv \bar{z}\left(t_{1}, t_{2}\right)$ represents the height of point $(x, y)$ in an irregular surface. The midpoint algorithm leads to a fractal self-affine surface, with fractal dimension $d_{f}=3-H$. In the corresponding iterative process, the set of points $(x, y)$ satisfying $\bar{z}(x, y)=\bar{z}_{0}$ constitutes a set of statistically self-similar curves, with fractal dimension $d_{f}=2-H$.

The algorithm starts with an square in the plane, with vertices placed at $\left(x_{A}, y_{A}\right),\left(x_{B}, y_{B}\right),\left(x_{C}, y_{C}\right)$, and $\left(x_{D}, y_{D}\right)$, and corresponding heights $\bar{z}_{A}, \bar{z}_{B}, \bar{z}_{C}$, and $\bar{z}_{D}$. The height, $\bar{z}_{E}$, of the point at the square centroid, $\left(x_{E}, y_{E}\right)$, is then vertically displaced from the average for $\bar{z}_{A}, \bar{z}_{B}, \bar{z}_{C}$, and $\bar{z}_{D}$ in a random way, just as in the one-dimensional case. In this way, we give raise to four triangles, formed by connecting $\bar{z}_{E}$ to the points $\bar{z}_{A}, \bar{z}_{B}, \bar{z}_{C}$, and $\bar{z}_{D}$. In the next generations, $g$, the same procedure is repeated by inserting a new point in the centroid of each existing triangle, the height $\bar{z}$ of which is taken as a random variable evaluated according to the prescription described above. The number of new sites increases as $g^{3}$, and the process is pushed forward until the a priori established maximal value for $n$ is reached.

In this work, any thermal effect due to high intensity electric currents is neglected. This is reasonable assumption based on recent results from experimental works ${ }^{13}$ indicating that current densities of $\sim 10^{8} \mathrm{~A} / \mathrm{cm}^{2}$ increase thermal resistivity in large roughness emitters. Since we use the FBM model with a relatively small value of $H$, we can be sure that the distance between peaks and valleys in the surface landscape is not very large and thermal effects are not important.

\section{B. Field emission calculations}

Field emission in metals is known to be essentially due to tunneling process. The transition probability for an electron to tunnel through the potential barrier and the number of incident electrons must then be estimated. In this way, evaluation of tunneling probabilities requires the determination of field emission current densities as a function of the electric field. Given the severe numerical difficulties implied in the evaluation of the electric-field intensity at the actual conductor irregular surface $\bar{z}(x, y)$, we approximate the actual emitting surface by an idealized $z(x, y)$. Here, $z$ represents the height of an equipotential surface $z_{\phi_{\varepsilon}}(x, y)$, associated to a very small nonzero value of the electric potential value $\phi_{\varepsilon}$, so that it lies very close to the actual surface where $\phi_{0}=0$. This technique has also the advantage to allow the possibility of mimicking surface emitter with different roughness. Details and reliability of this procedure have been given previously ${ }^{9}$ and then will not be discussed here. 
The electrostatic problem consists of numerically solving Laplace's equation,

$$
\nabla^{2} \phi=0 .
$$

with Dirichlet conditions at the boundaries and periodic conditions in the sidewalls. The upper boundary of the problem consists of a plane anode, which is set at a fixed potential value, $\phi^{A}$. We obtained results for $\phi^{A(\sigma)}=100+50(\sigma-1)$, with $\sigma=1, \ldots, 9$. For each value of $\phi^{A}$, the solution $\phi(\vec{r})$ was obtained by solving Eq. (2) in an equally spaced discretized grid, consisting of $L \times L \times M$ points $\overrightarrow{\mathbf{R}}=(\mathbf{i}, \mathbf{j}, \mathbf{k})$, with $\mathbf{i}, \mathbf{j}, \mathbf{k}$ $\in \mathbb{N}$. In order to cope with experimentally realizable conditions, we assume $L \gg M$. The coordinates $\vec{r}=(x, y, z)$ of the equipotential surfaces, $z_{\phi_{\varepsilon}}(x, y)$, which do not necessarily lye on the grid points, are obtained by an interpolation procedure. Similarly, the components of the electric field

$$
E^{\mu}(r)=-\nabla^{\mu} \phi(\vec{r}),
$$

where $\mu$ denotes the field component $(x, y$, or $z)$ at any point $\vec{r}$ of an equipotential surface, can be expressed in terms of the respective components $E^{\mu}(\overrightarrow{\mathbf{R}})$, at the eight grid points defined by the corners of the regular cube of unit volume within which $\vec{r}$ lies. Interpolation is then performed according to

$$
E^{\mu}(\vec{r})=\mathfrak{r}_{\text {norm }} \sum_{\ell, m, n=0}^{1} \frac{E^{\mu}(\mathbf{i}+\ell, \mathbf{j}+m, \mathbf{k}+n)}{\mathfrak{r}_{x_{\ell} y_{m} z_{n}}} .
$$

In Eq. (4), variables $\mathfrak{r}_{x_{\ell} y_{m} z_{n}}$ denote the distances from $\vec{r}$ to each of the cube corners, i.e.,

$$
\mathfrak{r}_{\ell, m, n}=\sqrt{\xi_{\ell}^{2}+\gamma_{m}^{2}+\zeta_{n}^{2}}
$$

where

$$
\xi=x-\mathbf{i}, \quad \gamma=y-\mathbf{j}, \quad \zeta=z-\mathbf{k},
$$

while

$$
\frac{1}{\mathfrak{r}_{\text {norm }}}=\sum_{\ell, m, n=0}^{1} \frac{1}{\mathfrak{r}_{x_{\ell} y_{m} z_{n}}} .
$$

From the definitions in Eqs. (5)-(7), it follows that -1 $<\xi_{\ell}, \gamma_{m}, \zeta_{n} \leq 1$, and $0 \leq \mathfrak{r}_{\ell, m, n} \leq \sqrt{3}$. It is also clear that if $\vec{r}$ coincides with any of the eight corners $\mathbf{R}$, i.e., when $\ell=m$ $=n=0$, both $\mathfrak{r}_{\text {norm }}$ and $\mathfrak{r}_{0,0,0}$ identically vanish, so that the contribution to $E^{\mu}(\vec{r})$ only comes from the field at $\overrightarrow{\mathbf{R}}$ [in this particular case, from $\overrightarrow{\mathbf{R}}=(i, j, k)]$. This limiting case indicates that Eq. (4) correctly averages the contribution from the eight vertices to the local field at $\vec{r}$.

To find the emission current density $J$, we make use of the FN approach, ${ }^{14}$ which has been systematically applied in problems where one has to optimize processes in small scale emitting devices. According to this framework, $J$ is related to the local electric-field intensity for an electron close to the Fermi surface trying to tunnel through a triangular potential barrier, the top of which is much higher than the Fermi energy.

The electron emission is described by the tunneling probability, which can be evaluated from the following one- dimensional, time-independent Schrödinger equation,

$$
-\frac{\hbar^{2}}{2 m} \frac{d^{2} \psi}{d x^{2}}+U(x) \psi=\epsilon \psi
$$

or

$$
\frac{d^{2} \psi}{d x^{2}}=\frac{2 m[U(x)-\epsilon]}{\hbar^{2}} \psi
$$

Assuming that $U(x)-\epsilon$ is independent of $x$ for a thin interval limited by $x$ and $x+d x$, the solution of Eq. (9) can be written as

$$
\psi(x+d x)=\psi(x) \exp (-k d x),
$$

where $k=\sqrt{2 m[U(x)-\epsilon]} / \hbar$. The negative sign in the exponent of Eq. (10) comes from the assumption that the electron moves from left to right. For a smooth variation in $U(x)$, the wave-function amplitude at $\psi\left(x_{2}\right)$ is related to $\psi\left(x_{1}\right)$ by the WKB approximation:

$$
\psi\left(x_{2}\right)=\psi\left(x_{1}\right) \exp \left(-\int_{x_{1}}^{x_{2}} \frac{\sqrt{2 m[U(x)-\epsilon]}}{\hbar} d x\right) .
$$

Since the transmission probability $T$ is expressed by

$$
T=\frac{\psi\left(x_{2}\right) \psi^{*}\left(x_{2}\right)}{\psi\left(x_{1}\right) \psi^{*}\left(x_{1}\right)},
$$

we finally arrive at the expression

$$
T=\exp \left(-\frac{2}{\hbar} \int_{x_{1}}^{x_{2}} \sqrt{2 m[U(x)-\epsilon]} d x\right) .
$$

To obtain the current density, $J$, we take into consideration that the electric field, $E^{\mu}$, caused by the anode potential controls the width of the potential barrier $\varphi-q E^{\mu} x$ through which an electron may tunnel, $\varphi$ being the material work function and $q$ the electron charge. By inserting the expression for this potential barrier into Eq. (13), it follows quite easily that the "cold" current density, $J$, is proportional to the mean value of the transmission coefficient $\langle T\rangle$ which, for the case of a three-dimensional configuration, becomes

$$
J=\frac{q^{3}(\bar{E})^{2}}{16 \pi^{2} \hbar \varphi} \exp \left(-\frac{\sqrt{32 m} \varphi^{3 / 2}}{3 q \hbar \bar{E}}\right)=\frac{K(\bar{E})^{2}}{\varphi} \exp \left(-\frac{N \varphi^{3 / 2}}{\bar{E}}\right),
$$

where $\bar{E}$ is the resulting local field following from the components $E_{\phi_{(\sigma, \epsilon)}}^{\mu}$, and parameters $K$ and $N$ have obvious definitions. It is usual to define the field amplification factor, $\gamma$, as $\bar{E}=\gamma E_{0}$, where $E_{0}=\phi^{A(\sigma)} / M$ is the uniform electric field generated by two parallel plates separated a distance $M$. This parameter gives the enhancement of the field taking place at sharp tips. Moreover, this factor is related to the slopes in the Fowler-Nordheim plots described in Sec. III.

\section{RESULTS AND DISCUSSION}

In the present paper, we intend to model rough tungsten surfaces, for which the work function has a value of $\varphi$ 


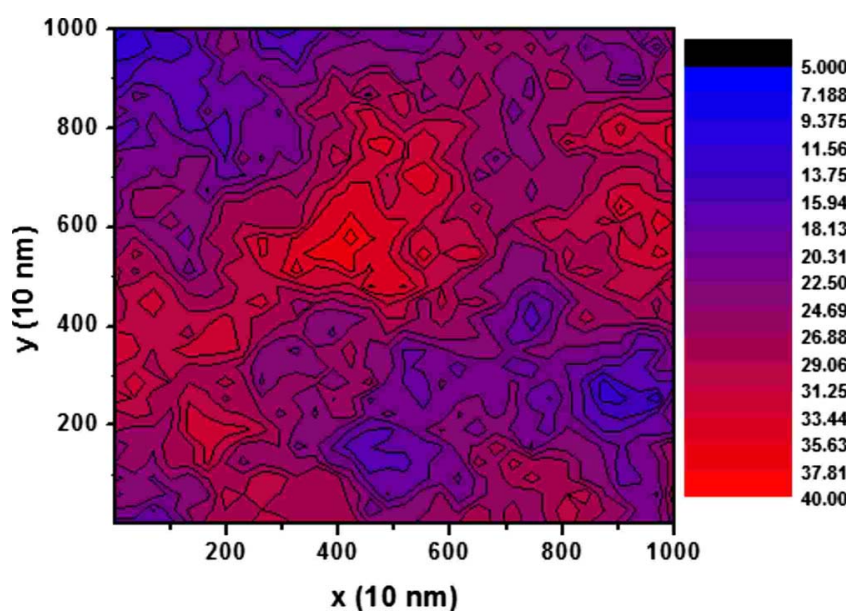

FIG. 1. (Color online) Contour lines of an irregular surface generated by the FBM algorithm for a Hurst exponent of $H=0.3$.

$=4.5 \mathrm{eV}$. Values of parameters $K$ and $N$ defined in Eq. (14) were conveniently chosen so that $J$ comes expressed in $\mathrm{A} / \mathrm{cm}^{2}$ units. ${ }^{15}$ Tungsten surfaces have been used for the production of cathodes in high-vacuum lines and then they have been thoroughly tested by microscopy techniques, such as scanning field emission microscopy. ${ }^{16}$ Several experimental works discussing the use of different materials, including tungsten, suggest that low intensity fields are able to trigger the emission process, provided that the surface is prepared with an irregular geometry in order to profit from the field gain factor that has been measured in association with it. ${ }^{15,17}$

In Fig. 1, we illustrate an irregular surface produced by the described FBM algorithm for a Hurst exponent $H=0.3$. Figure 2 shows the behavior of the roughness, $W(h)$, as a function of the scale size, $h$, for different approximations of the emitting surface. According to the discussion presented in Sec. II, we approximate the emitting surface by equipotential surfaces evaluated at very low values of the potential at the following values: $\phi_{\epsilon}=\left(\phi_{1}, \ldots, \phi_{4}\right)$, where $\phi_{4}=2 \phi_{3}$

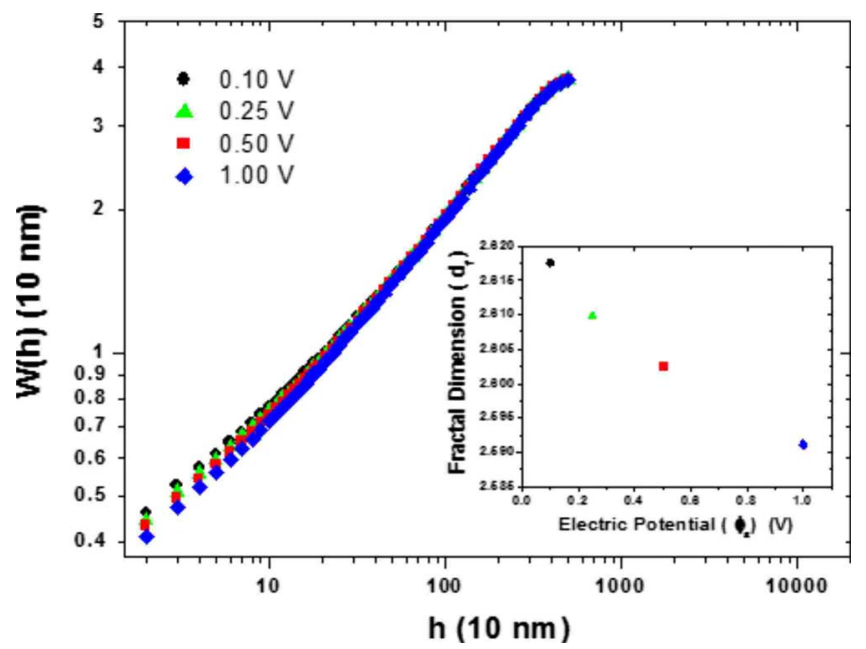

FIG. 2. (Color online) Roughness of the equipotential surfaces, $W(h)$ calculated near the actual surface showed in Fig. 1 as a function of the scale size, $h$. The fractal dimension, $d_{f}$, related to equipotential surfaces is also given in the inset.
$=4 \phi_{2}=10 \phi_{1}=1.00 \mathrm{~V}$. The roughness $\mathrm{W}$, measured in units of dozens of nanometers, is estimated by the average value of the dispersion of the height $z$ by the rms method. It is possible to notice that the difference in the small scale behavior of $W$ leads to different values of the corresponding roughness exponent $H_{\phi_{\epsilon}}$, and fractal dimensions $d_{f_{\phi \epsilon}}$. These follow from the evaluation of the slope of the curves that has been performed in the scale interval $[20,200] \mathrm{nm}$.

Let us discuss next the dependence of $W(h)$ and $d_{f_{\phi_{\epsilon}}}$ with respect to the emitting properties of the material. Taking into consideration that these magnitudes directly reflect the surface topology, our results suggest strong differences on the local values of $J$ when different approximations for the potentials $\phi_{1}$ and $\phi_{4}$ are considered. Despite the fact that the global maxima, $W\left(h_{\max }\right)$, are quite close (see Fig. 2), the resulting current densities are quite different. For instance, when the anode potential is fixed at a value of $\phi^{A(\sigma=2)}$ $=150 \mathrm{~V}$, we obtain $J_{\phi_{1}} / J_{\phi_{4}} \approx 10^{2}$. Since the $\phi_{1}$ equipotential lies much closer to the actual surface, this result shows a strong connection between the local charge density and the local irregularities. The results also reveal the presence of high local values of $J$ for any $\phi_{\epsilon}$, something that is related to the scale-invariance properties of the emitting surface.

This claim is also supported by the curves for the distribution of local-field intensities, $\rho(\bar{E})$, for the same fixed value of the anode potential and the four different potential values of the approximate emitting surface. This is shown in Fig. 3(a), where the distribution curves display significant changes in the small values of the field intensity. The changes indicate: (a) an increase in the frequency of very small values of $\bar{E}$, causing a displacement on the peak to the left side; (b) a decrease in the distribution maximum, leading to an increase in the dispersion. These two features are progressively enhanced as the approximate emitting surface gets closer to the actual one, this corresponding to an increase in the surface roughness. The increase in the number of points with small field intensity when the roughness increases results from the fact that the points lying between two sharp peaks have large field components $E_{\phi_{(\sigma, \epsilon)}^{\mu}}^{\mu}$ in the horizontal plane which, with high probability, cancel each other, leading to small values of $\bar{E}$. This has a direct influence on the mean value of the electric field, which continuously decreases as $\phi_{\epsilon} \rightarrow 0$. This result is in agreement with the interpretation given in a previous paper. 9 Finally, let us remark that we have also presented superimposed in Fig. 3(a) a typical experimental value of the turn on field $\left(\sim 8 \times 10^{6} \mathrm{~V} / \mathrm{cm}\right)$ for tungsten surfaces ${ }^{18}$ in order to make clear that the intensities obtained in this work are compatible with it. To compare the results corresponding to rough and smooth surfaces, we have also plotted in Fig. 3(a) the value of the constant electric field produced between flat parallel anode and cathode (full gray vertical line), and the distribution $\rho(\bar{E})$ for a very smooth FBM surface (dashed line). In both cases, we take the corresponding anode-cathode distance as the average distance between the rough plane and anode, as defined in Ref. 9.

We have also evaluated the distribution of the local roughness, $\rho\left[W^{l}(h)\right]$ and its dependence on the characteristics of 

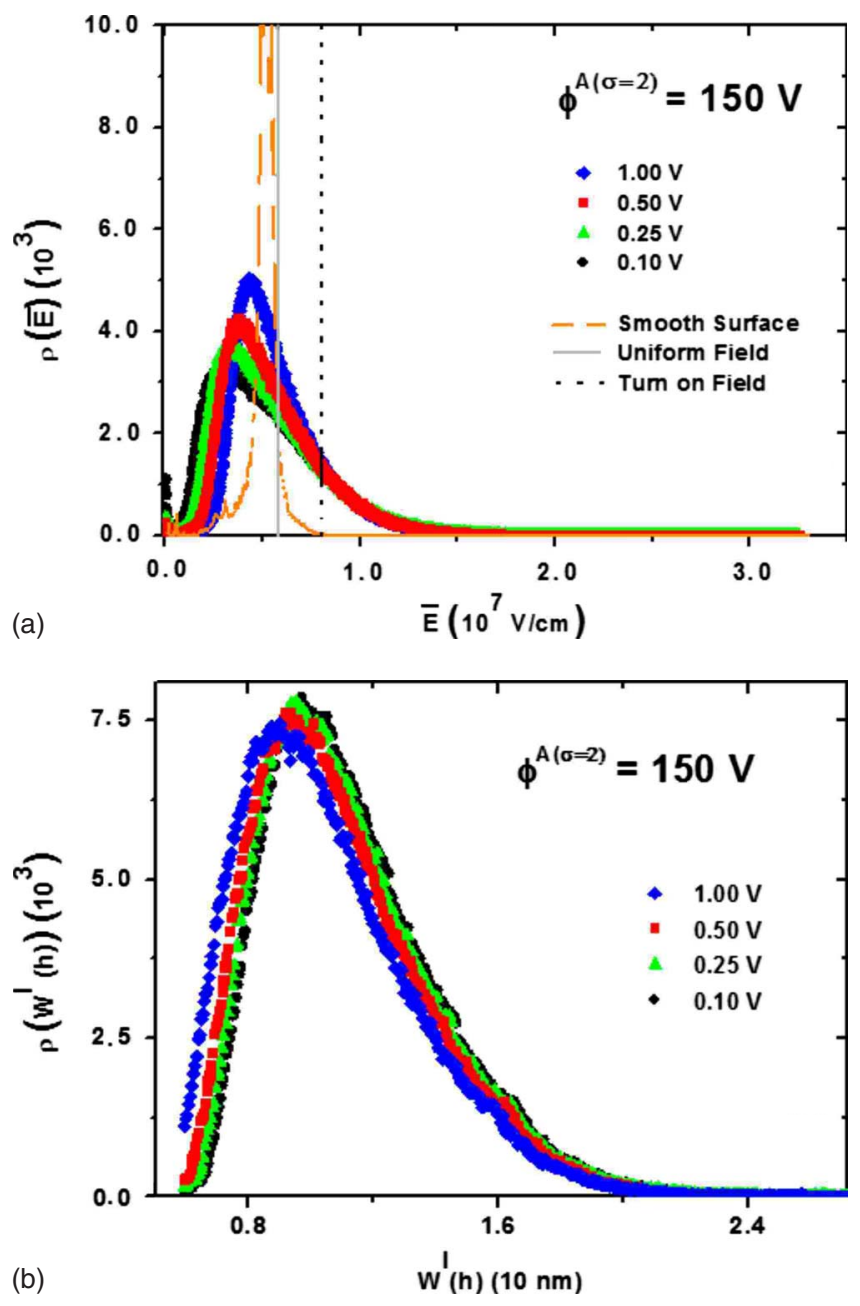

FIG. 3. (Color online) (a) Distribution, $\rho(\bar{E})$, of the local electric-field intensities, $\bar{E}$, for different separation approximations of the emitting cathode by equipotential surfaces defined by $\left(\phi_{1}, \ldots, \phi_{4}\right)$. Two vertical lines indicate the position of the uniform electric field produced by the cathode potential between two parallel plane plates (full line) and the turn on field for the used rough surface (dotted line). In the first case, the distance between the plates is evaluated using the average distance between the anode and local cathode height $z(x, y)$ (see Ref. 9 for details). The same consideration is applied to a very smooth FBM emitter, whose $\rho(\bar{E})$ distribution is also showed (dotted line). (b) Distribution, $\rho\left[W^{l}(h)\right]$, of the local roughness, $W^{l}(h)$, for the same approximate emitting surfaces.

the emitting surface. The results are shown in Fig. 3(b), where the distribution curves for the same potential values of the emitting surface as in Fig. 3(a) are given. The local roughness was evaluated with the algorithm described in Ref. 19 which amounts to perform an scale-invariance analysis on small square windows of size $h=20 \mathrm{~nm}$ around each point $(x, y)$. The local roughness is then estimated by the dispersion

$$
W^{l}(h)=\frac{1}{n_{h}} \sum_{i=1}^{n_{h}} \sqrt{\frac{1}{m_{i}} \sum_{\delta \in h}\left[z_{\delta}(x, y)-\langle z\rangle\right]^{2}},
$$

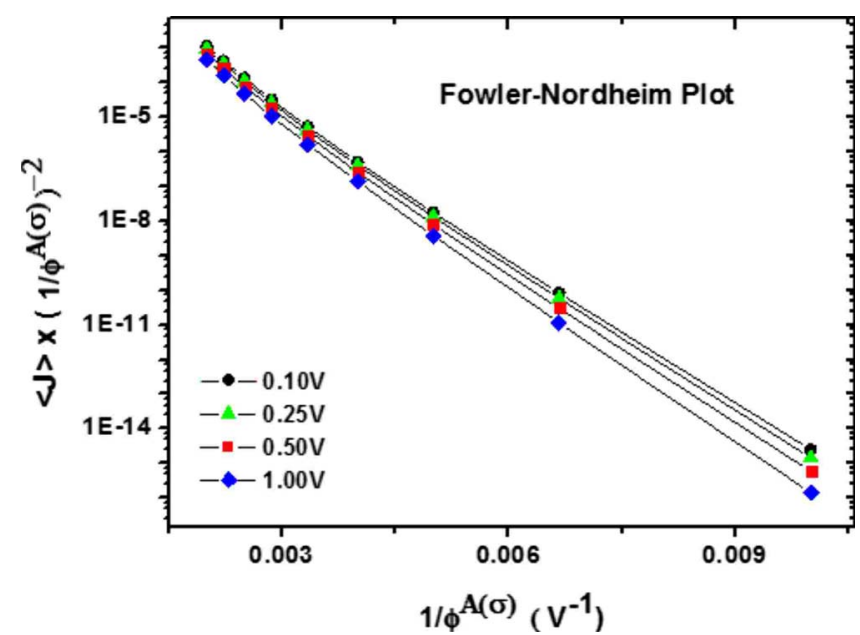

FIG. 4. (Color online) Fowler-Nordheim plot, representing how the average current density depends on the anode electric potential $\phi^{A(\sigma)}$. Circles, triangles, squares, and diamonds represent the emission surfaces defined by $\phi_{\epsilon}=\phi_{1}, \phi_{2}, \phi_{3}$, and $\phi_{4}$, respectively.

where $n_{h}, m_{i}$, and $\langle z\rangle$ indicate, respectively, the total number of square windows of side $h$, the number of points inside the $i$ th window, and the average height of the points in the considered window.

Comparison between Figs. 3(a) and 3(b) reveals a correlation between the local roughness and the morphology of the equipotential surfaces used to approximate the emitting cathode. In the $\phi_{\epsilon} \rightarrow 0$ limit, a decrease on the frequency of points in the small local roughness $W^{l}(h)$, (i.e., in the interval $[5,7] \mathrm{nm})$ is observed, together with an increase in the frequency for larger values of $W^{l}(h)$.

The dependence of emission properties on $W^{l}(h)$ is also supported by the analysis of the Fowler plots (FPs), which relates the average emission current, $\langle J\rangle$, with respect to the anode potential. The averaged value $\langle J\rangle$ for an emitting surface defined by $\phi_{\epsilon}$ and anode potential $\phi^{A(\sigma)}$ is evaluated by summing over the local current density $J=J_{n},(n$ $=1,2, \ldots, L \times L)$, which is given by Eq. (14). Therefore, we obtain

$$
\langle J\rangle=\frac{1}{L \times L} \sum_{n=1}^{L \times L} J_{n} .
$$

The results are shown in Fig. 4, where two different regimes, distinguishing the high and low potential regions, are seen. The first one is characterized by a linear dependence, suggesting the existence of a threshold value after which electrons are emitted by any site on the surface. In the low potential region the linear dependence is lost, indicating that the number of emitting points reduces and is dependent of the material local roughness. The enhanced electronic emission by larger roughness surfaces is independent of the anode potential, so that $\left\langle J_{\phi_{1}}\right\rangle>\left\langle J_{\phi_{2}}\right\rangle>\left\langle J_{\phi_{3}}\right\rangle>\left\langle J_{\phi_{4}}\right\rangle$ for any value of $\phi^{A(\sigma)}$. This shows that the emission properties depend essentially on the own features of the emitting surface. In this way, it is possible to observe that the deviation from the linear regime of the Fowler plot curves, for low values of the anode potential, is more intense. Such result clearly indicates a cor- 


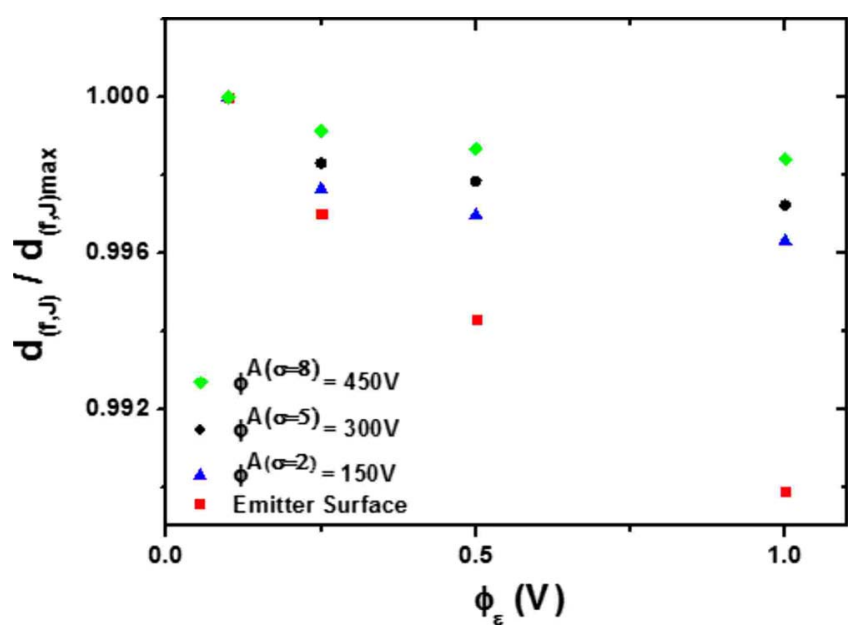

FIG. 5. (Color online) Normalized fractal dimension of the emission current surfaces $z_{J\left(\phi_{\epsilon A}\right)}$ : triangles, circles, and diamonds correspond to $\phi^{A}=150,300$, and $450 \mathrm{~V}$, respectively. For comparison, we also plot (squares) here the normalized fractal dimension of the emission surfaces $z_{\phi_{\epsilon}}$, obtained from the data in the inset of Fig. 2.

relation between the irregularities of the emitting surface and its current-density intensity at low field. Furthermore, when the surface roughness increases, the differences between the corresponding values of $J$ are less and less dependent on the value of the anode potential. It is important to recall that, for the system size $L \times L \times M$ and values of $\left\{\phi^{A(\sigma)}\right\}$ used herein, the slope of curve $\left\langle J_{\phi_{\epsilon}}\right\rangle /\left(\phi^{A(\sigma)}\right)^{2}$ as a function of $1 / \phi^{A(\sigma)}$ has not yet reached the limit value. Indeed, the number of void local current densities, $J_{\phi}$, contributing to the average value $\left\langle J_{\phi}\right\rangle$ depend on $\phi^{A(\sigma)}$ differently. This can be made clear by following the value of $J_{\phi_{\epsilon}}$ for some small surface patches. So, the local slope of the FPs showed in Fig. 4 have a clear dependence with the applied electric field, indicating more effective surface emission in regions with higher voltages. At sufficiently high voltages, when all surface is emitting, the slope becomes constant and the value of emitting area saturate.

Let us work out now one more evidence of the relationship existing between the emission properties of a cathode and the fractal dimension of its surface. For this purpose, we define an artificial emission current surface $z_{J\left(\phi_{\epsilon, A}\right)}=f(x, y)$, the height of which is equal to the current density produced by the corresponding emission surface $\phi_{\epsilon}$ and anode potential $\phi^{A}$. This definition makes it possible to evaluate the fractal dimension of the virtual surface $J(x, y)$, so that we can check whether $z(x, y)$ and $J(x, y)$ have similar properties. In this way it is possible to repeat the fractal analysis for the surface $z_{J\left(\phi_{\epsilon, A}\right)}=f(x, y)$. The corresponding results are shown in Fig. 5, where the behavior of the fractal dimension $d_{f, J}$, normalized to its largest value $d_{(f, J)_{\max }}$ for each individual surface $z_{J\left(\phi_{\epsilon, A}\right)}=f(x, y)$, is displayed. For the sake of comparison, we also include in the figure the corresponding values of the normalized fractal dimension of the emission surfaces $d_{f} / d_{\max f}$. It can be noticed that by increasing the value of the anode potential $\phi^{A(\sigma)}$, the rate between $d_{f, J} / d_{(f, J)_{\max }}$ increases monotonically. For large fixed value of $\phi^{A(\sigma)}$ all current sur- faces $z_{J\left(\phi_{\epsilon, A}\right)}$ have similar fractal dimensions, so that the points for $d_{f, J} / d_{(f, J)}$ as function of $\epsilon$ shows plateaus, while the corresponding ratio for the equipotential emitting surfaces decreases in a quite pronounced way. If we compare Figs. 4 and 5, we notice that, for large values of the potential, all curves in the Fowler plot for different emitting surfaces have similar slopes. This correlates with the existence of the plateau for the current surfaces $z_{J\left(\phi_{\epsilon, A}\right)}$ in Fig. 5. As the value of $\phi^{A(\sigma)}$ decreases, the FPs have different slopes, causing the plateau to be less and less pronounced. These results for the fractal dimension of $z_{J\left(\phi_{\epsilon, A}\right)}$ suggest that it may exist a dependence with the emitting surface since the behavior is similar to that of the fractal dimension of the emitting surface $\phi_{\epsilon}$. This leads to the existence of a direct correlation between the fractal dimension of $z_{J\left(\phi_{\epsilon, A}\right)}$ and the local-field amplification factor. In the present work, this factor is defined as the ratio between the local field of a point in a rough profile $\bar{E}(\vec{r})$ and the corresponding uniform field resulting from a potential difference $\phi^{A(\sigma)}$ and the distance $M$, as described at the end of Sec. II. This factor can be experimentally measured from the lifetime of voltage pulses $t_{p}$ (i.e., the decay time $t_{d}$ ), which are able to cause damages in the emitting surface structure. $^{20}$

Finally, we would like to stress that from the series of results presented here it is quite evident that, at low values of electric field, a strong correlation between the emitting properties and the fractal dimension of the surface is found. In designing emitters for particular purposes, self-similar microirregularities at a given scale interval then plays an important role.

\section{SUMMARY AND CONCLUSIONS}

In this work we investigated how the geometrical features of irregular surfaces are related to cold emission properties of conducting materials. Our analysis requires, in first place, the numerical integration of Laplace equation in a threedimensional region bounded by a plane and a rough surface. One of these surfaces is a rough surface mimicking the emitting cathode, while a far away plane aims to describe the corresponding anode. Several features of our results agree well with experimental reports for electron emission by an irregular tungsten surface subject to an electric field 8 $\times 10^{6} \mathrm{~V} / \mathrm{cm}$. The results reported here also allow to estimate to which extension the electronic emission is influenced by the surface roughness, at low values of the emission field.

We believe that these results can help to explain some differences between theoretical calculations with smooth geometries and experimental studies, which predict high values of the field amplification factor and very small values of effective emitting area. We have shown that our results suggest that the fractal dimension may be related to the field amplification factor, while the total emission current is determined by the roughness of the surface. Then, in general terms, the emitting properties are related to the fractal structure of the surface emitter in a twofold way: (i) the existence of protuberances with respect to a average ground height and (ii) the contribution to an effective-field amplification produced by irregularities at nanometric scales. 


\section{ACKNOWLEDGMENTS}

This work has been supported by the Ministerio de Educación y Ciencia (Spain) under Projects No. MTM200615533 and No. CONSOLIDER 2006-32 (i-Math), Comunidad de Madrid under Project No. S-0505/ESP-0158
(SIMUMAT), and CNPq under Grant No. 306052/2007-5 (Brazil). T.A.d.A. gratefully acknowledges a doctoral grant from AECI (Spain). The authors also acknowledge C. M. C. de Castilho, J. G. V. Miranda, and F. B. Mota for their comments that have contributed to improve the paper. *t.albuquerque@uam.es

†f.borondo@uam.es

†rosamaria.benito@upm.es

\$randrade@ufba.br

${ }^{1}$ O. M. Bulashenko and J. M. Rubí, Nanotechnology 14, 200 (2003).

${ }^{2}$ W. Zhu, C. Bower, G. P. Kochanski, and S. Jin, Solid-State Electron. 45, 921 (2001).

${ }^{3}$ V. A. Solntsev and A. N. Rodionov, Solid-State Electron. 45, 853 (2001).

${ }^{4}$ T. Habermann, A. Gohl, D. Nau, M. Wedel, G. Muller, M. Christ, M. Schreck, and B. Stritzker, Proceedings of the 10th International Vacuum Microelectronics Conference (IVMC), 1997, p. 156.

${ }^{5}$ J. M. Bonard, K. A. Dean, B. F. Coll, and C. Klinke, Phys. Rev. Lett. 89, 197602 (2002).

${ }^{6}$ M. Bianchetti, P. Buonsante, F. Ginelli, A. Lorenzone, H. E. Roman, and R. A. Broglia, The 13th International Winterschool on electronic properties of novels materials-science and technology of molecular nanostructures, AIP Conf. Proc. No. 486 (AIP, New York, 1999), p. 448.

${ }^{7}$ M. Araidai and K. Watanabe, Appl. Surf. Sci. 237, 483 (2004).

${ }^{8}$ T. A. de Assis, F. B. Mota, J. G. V. Miranda, and R. F. S. Andrade, H. de O. Dias Filho, and C. M. C. de Castilho, J. Phys.:
Condens. Matter 18, 3393 (2006).

${ }^{9}$ T. A. de Assis, F. B. Mota, J. G. V. Miranda, R. F. S. Andrade, and C. M. C. de Castilho, J. Phys.: Condens. Matter 19, 476215 (2007).

${ }^{10}$ J. C. Russ, Fractal Surfaces (Plenum, New York, 1994).

${ }^{11}$ J. Feder, Fractals (Plenum, New York, 1988).

${ }^{12}$ G. Miller, Comput. Graph. 20, 39 (1986).

${ }^{13}$ K. Yeong and J. Thonga, J. Appl. Phys. 99, 104903 (2006).

${ }^{14}$ R. H. Fowler and L. W. Nordheim, Proc. R. Soc. London, Ser. A 117, 173 (1928).

${ }^{15}$ Huang Kai, P. Qingtao, Y. Feng, N. Shibi, and H. Deyan, Appl. Surf. Sci. 253, 8923 (2007).

${ }^{16}$ S. Sendecki and B. Barwinski, Appl. Surf. Sci. 134, 243 (1998).

${ }^{17}$ P. D. Rack, J. Li, P. Ostdiek, J. Peterson, L. Linh, and T. Rogelstad, Proceedings of the 14th Biennial Industry Microelectronics Symposium, 2001, p. 140.

${ }^{18}$ Hsieh Chin-Tang and T. Jyh-Ming, Chem. Phys. Lett. 413, 84 (2005).

${ }^{19}$ E. Vidal Vázquez, J. G. V. Miranda, and A. P. González, Ecol. Modell. 182, 337 (2005).

${ }^{20}$ A. A. Emelyanov, Proceedings of the 17th International Symposium on Discharges and Electric Insulation in Vac., 1996, Vol. 1, p. 32 . 